\title{
ALK2 mutation in a patient with Down's syndrome and a congenital heart defect
}

\author{
Irene C Joziasse ${ }^{1,8}$, Kelly A Smith ${ }^{2,3,8}$, Sonja Chocron ${ }^{2,3}$, Maarten van Dinther ${ }^{4}$, Victor Guryev ${ }^{2}$, \\ Jasper J van de Smagt ${ }^{5}$, Edwin Cuppen ${ }^{2,5}$, Peter ten Dijke ${ }^{4}$, Barbara JM Mulder ${ }^{3,6}$, Cheryl L Maslen ${ }^{7}$, \\ Benjamin Reshey ${ }^{7}$, Pieter A Doevendans ${ }^{1}$ and Jeroen Bakkers ${ }^{\star 2,3}$
}

\begin{abstract}
Down's syndrome (DS), resulting from an additional copy of chromosome 21 (trisomy 21), is frequently associated with congenital heart defects (CHDs). Although the increased dosage of chromosome 21 sequences is likely to be part of the etiology of cardiac defects, only a proportion of DS patients exhibit a congenital heart defect (birth prevalence $40-60 \%$ ). Through a large-candidate gene-sequencing screen in patients with atrioventricular septal defects, substitutions were identified in bone morphogenetic protein (BMP) type I receptor $A L K 2$ and two other genes in a patient with DS and a primum-type atrial septal defect. Structural modeling of the cytoplasmic domain of the ALK2 receptor suggests that H286 is in close proximity to the nucleotide-binding site of the kinase domain. We investigated whether this p.His286Asp substitution altered ALK2 function by using both in vitro as well as in vivo assays. The p.His286Asp variant demonstrated impaired functional activity as measured by BMP-specific transcriptional response assays. Furthermore, mild dominant-interfering activity was observed in vivo compared with wild-type $A L K 2$ as determined by RNA injection into zebrafish embryos. These data indicate that in the context of a DS background, ALK2-mediated reduction of BMP signaling may contribute to CHDs.
\end{abstract}

European Journal of Human Genetics (2011) 19, 389-393; doi:10.1038/ejhg.2010.224; published online 19 January 2011

Keywords: Down's syndrome; congenital heart defects; gene mutation

\section{INTRODUCTION}

Down's syndrome (DS) is the most frequent cause of mental retardation, most often caused by trisomy 21 (in approximately $95 \%$ of cases). DS occurs in about one in 700-1000 live births. ${ }^{1-3}$ The syndrome is characterized by well-defined and distinctive phenotypic features including characteristic facies, minor limb anomalies, hypotonia and growth retardation. ${ }^{4}$ Although all individuals with DS manifest these phenotypes, there is significant phenotypic variability between such individuals. ${ }^{5}$ In conjunction with these phenotypic traits, DS individuals suffer from a higher incidence of several conditions, including CHDs. ${ }^{5}$

The (birth) prevalence of CHDs in DS is about $40-60 \%$, most frequently atrioventricular septal defects and ventricular septal defects. ${ }^{6-8}$ Efforts have been made to determine the critical chromosomal region for specific phenotypic features of DS by deletion mapping and characterization of patients with partial trisomy $21.4,9,10$ One potential candidate gene for these trisomy 21-related CHDs is collagen type VI. However, the evidence for the involvement of collagen type VI has, to date, been restricted to correlative expression pattern analysis. ${ }^{11,12}$

Although this susceptibility and variability of the DS phenotype is widely accepted, the factors contributing to this variability have not been established. It is postulated that variations in gene dosage of chromosome 21, environmental factors and genetic modifications not linked to chromosome 21 account for this variability, and it is likely that contributions from each of these elements are involved. Genetic imbalance caused by the presence of an extra copy of chromosome 21 will seriously disrupt one or more developmental pathways. In addition, the presence of non-chromosome 21 loci variation may predispose for a heart defect. To date, CRELD1 (3p25.1) is the only non-chromosome 21 gene that has been reported to associate with DS-related CHDs. ${ }^{13}$

We have previously reported mutations in $A L K 2$, a type I receptor for BMPs, in patients with endocardial cushion-associated CHDs. One of these variants identified has a dominant-interfering effect on BMP signaling and is associated with primum-type atrial septal defect (ASD) type $\mathrm{I}^{14}$ Here we report an additional $A L K 2$ variant that was identified in a patient with trisomy 21 and a primum-type ASD (ASD type I). In addition to the $A L K 2$ variant, two other variations were detected in another type I BMP receptor gene, $A L K 3$, and in the epidermal growth factor receptor family, ErbB3. Using both in vitro and in vivo assays, we show that the ALK2 variant has reduced BMPinductive capacity and a mild dominant-interfering effect on BMP signaling. This effect on BMP signaling from the ALK2 variation in

\footnotetext{
${ }^{1}$ Department of Cardiology, University Medical Center Utrecht, Utrecht, The Netherlands; ${ }^{2}$ Cardiac Development and Genetics, Hubrecht Institute and University Medical Center Utrecht, Utrecht, The Netherlands; ${ }^{3}$ Interuniversity Cardiology Institute of the Netherlands, Utrecht, The Netherlands; ${ }^{4}$ Department of Molecular Cell Biology, Leiden University Medical Center and Center for Biomedical Genetics, Leiden, The Netherlands; ${ }^{5}$ Department of Medical Genetics, University Medical Center Utrecht, Utrecht, The Netherlands; ${ }^{6}$ Department of Cardiology Academic Medical Center, Amsterdam, The Netherlands; ${ }^{7}$ Division of Cardiovascular Medicine, Oregon Health \& Science University, Portland, OR, USA

*Correspondence: Associate Professor J Bakkers, Department of Cardiac Development and Genetics, Hubrecht Institute and University Medical Center, Utrecht 3508, Netherlands. Tel: +31 30 2121892; Fax: +31 30 2121801. E-mail: j.bakkers@hubrecht.eu

8 These authors contributed equally to this work.

Received 6 April 2010; revised 14 September 2010; accepted 19 October 2010; published online 19 January 2011
} 
conjunction with the additional variations identified in this patient is likely to contribute to the manifestation of a CHD in this individual.

\section{MATERIALS AND METHODS}

This study was prospectively reviewed and approved by the Local Research Ethics Committee of the University Medical Center Utrecht and the Academic Medical Center, Amsterdam, The Netherlands.

\section{Genetic material and clinical evaluation}

The patient described in this study was identified in a large-scale candidate gene sequencing screen as previously described. ${ }^{14}$ In total, nine candidate genes were sequenced in this patient (ALK2, ALK3, EGFR, GATA4, HAS2, UGDH, ERBB2, $E R B B 3$ and CRELD1). Patient material was acquired from the CONCOR (CONgenital CORvitia) database. ${ }^{15}$ DNA material was obtained from the patient and first-degree relatives by buccal swabs. Family members were clinically evaluated and individuals testing positive for the $A L K 2, E R B B 3$ and $A L K 3$ variant were examined by echocardiography. DNA material from patients with non-cardiac-related diseases (Fragile X $(n=170)$, male infertility $(n=90)$ and Rett syndrome $(n=90)$ ) was used for controls. Controls were sourced from the Dutch population, that comprises $80 \%$ of individuals of European ancestry (according to the Central Bureau of Statistics, The Netherlands, for 2008).

\section{Detection of variants}

PCR primers (sequence available upon request) for amplification were designed using an in-house developed management system (http://limstill.niob.knaw.nl), which was also used for the semi-automated identification and classification of mutations. ${ }^{16}$ Selected genes were analyzed by dideoxy sequencing of PCRamplified exonic fragments.

\section{Cell lines and transfections}

Bovine aortic endothelial cells ${ }^{17}$ were used for luciferase assays and $\cos 7$ cells were used for construct expression. All transfections were performed using Lipofectamine (Invitrogen, Carlsbad, CA, USA) according to manufacturer's instructions.

\section{Constructs, luciferase assay and kinase activity assays}

Expression constructs encoding full-length human ALK2 were cloned into the pcDNA3 vector as previously described, ${ }^{18}$ and mutant variants were generated by site-directed mutagenesis using a QuikChange kit (Stratagene, La Jolla, CA, USA). Inserts were fully sequenced and recloned into the original parental vector or the pCS2+ vector (for mRNA synthesis) to ensure against additional mutations. The luciferase assay was performed as previously described. ${ }^{19}$ This included an injection with vector only or in combination with a short

a

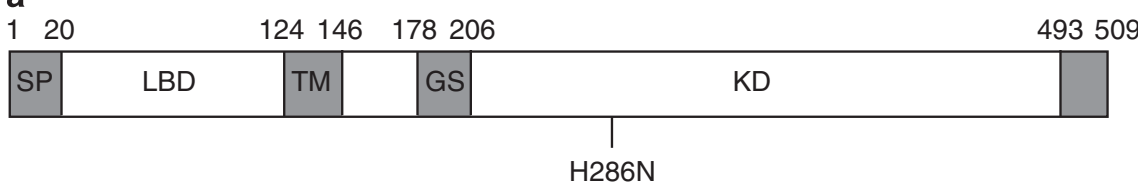

b

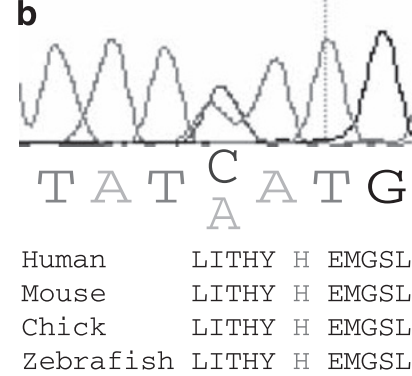

d

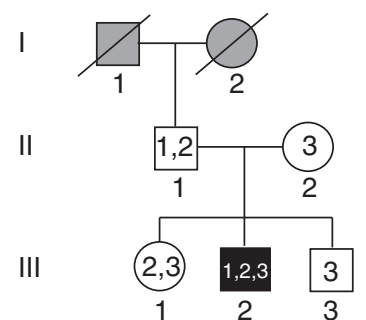

C
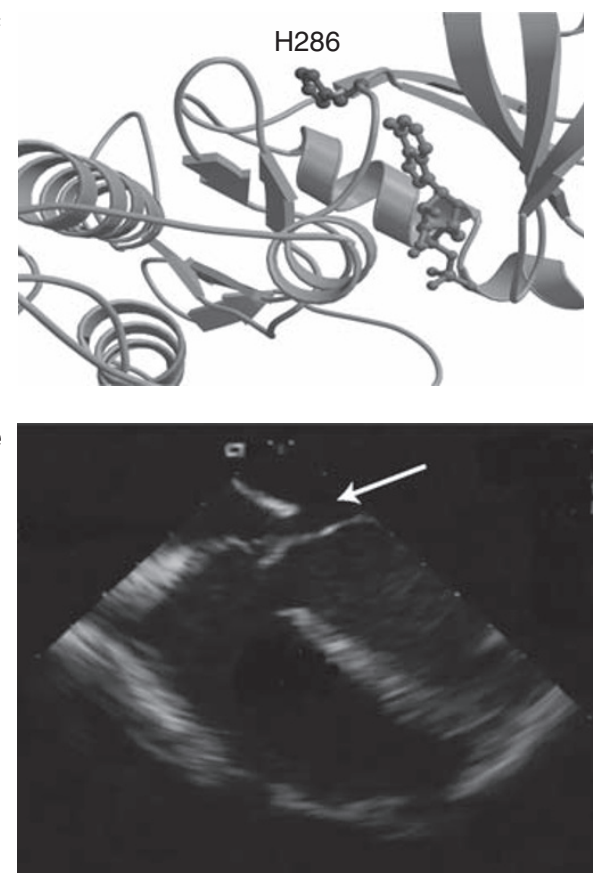

Figure 1 Identification of ALK2 variant in DS. (a) Schematic representation of the ALK2 protein and structural domains: signal peptide (SP), ligand-binding domain (LBD), transmembrane domain (TM), GS domain (GS) and kinase domain (KD). Arrows indicate approximate locations of p.His286Asp in ALK2. (b) Sequencing chromatogram with alignment below (aligned by ClustalW) showing the ALK2 p.His286Asp variant in genomic DNA from the proband with DS and a primum-type atrial septal defect. The protein alignment shows strong conservation between species of the ALK2 p. His286Asp residue. (c) TGF $\beta R$ I (pdb entry 1PY5) as a model for ALK2, showing H268 in red, ADP in blue and the $\beta$-sheet in yellow. (d) Family pedigree of proband (III:2). Only the father, without DS, was in possession of the ALK2 variant and did not have a congenital heart defect by echocardiography. Carriers of variants are depicted with a number: 1(ALK2 p.His286Asp), 2 (ALK3 p.Glu415Lys) or 3 (ErbB3 p.Thr1169lle), and individuals with or without CHDs are shaded black or white, respectively. Gray coloration indicates the genotype or phenotype was unable to be determined. Deceased individuals are annotated by a diagonal strikethrough. (e) Echocardiogram of proband carrying the p.His286Asp variant (III:2) before surgery, demonstrating a large primum-type atrial septal defect (arrow) and overriding AV valve. 
hairpin RNA, the latter to knockdown endogenous bovine ALK2 but not vector-based human ALK2, as described previously. ${ }^{14}$

\section{Zebrafish lines, mRNA synthesis and injections}

Wild-type fish were kept under standard conditions as previously described. ${ }^{20}$ pCS2+ ALK2 constructs were linearized with NotI enzyme, and capped mRNA was prepared with the Message Machine kit (Ambion, Austin, TX, USA). mRNA was diluted to appropriate concentration in nuclease-free water and $1 \mathrm{nl}$ (see ref. 20) per embryo injected at the 1-2-cell stage. Embryos were imaged live and mounted in methylcellulose.

\section{Statistical analysis}

Results are expressed as mean \pm SEM. Statistical significance was determined by one-way analysis of variance, followed by a Bonferroni $t$-test using the program SigmaStat version 2.03 (Jandel Scientific, San Rafael, CA, USA).

\section{RESULTS}

We recently performed a screen for the detection of cSNPs in patients with CHDs arising from improper endocardial cushion formation. ${ }^{14}$ During this screen we identified a patient with DS in possession of compound cSNPs in ALK2 that resulted in a His286Asp substitution ( $A L K 2$ p.His286Asp) (Figure 1b); the ALK3 cSNP caused a Glu414Lys substitution (ALK3 p.Glu414Lys), while the cSNP in ERBB3 resulted in a Thr1169Ile substitution (ERBB3 p.Thr1169Ile) (Supplementary Figure 1). These cSNPs were analyzed with the prediction tools PolyPhen $^{21,22}$ and SIFT $^{23}$ (Table 1). Only ALK2 p.His286Asp was predicted to damage protein function in both models and was not detected in 350 controls of the Dutch population. The coding regions of a total of nine genes were sequenced in this individual, including CRELD1, and no other CSNPs were identified.

This patient with DS was diagnosed with a primum-type atrial septal defect with a cleft mitral valve and the coronary sinus draining into the left atrium (Figure 1e). Sequencing of DNA from peripheral blood lymphocytes confirmed the three cSNPs in this individual (Figure 1d). To determine heritability and segregation of the cSNPs, we screened all first-degree relatives of the proband for the presence of each substitution (Figure 1d). The father of the proband was found to possess both the ALK2 p.His286Asp and the ALK3 p.Glu414Lys variants, whereas the mother was a carrier of the ERBB3 p.Thr1169Ile variant. Of the proband's two siblings, only the sister carried the $A L K 3$ p.Glu414Lys and the ERBB3 p.Thr1169Ile variants, whereas the brother was negative for the three cSNPs. None of these relatives were found to have a CHD by cardiac echocardiography.

We utilized our previous experience in functionally characterizing ALK2 variants to investigate the ALK2 p.His286Asp variant. Structural modeling of the cytoplasmic domain of the ALK2 receptor demonstrated that $\mathrm{H} 286$ resides within the ATP binding pocket of the kinase domain, indicating that a missense substitution at this residue may impair receptor activity (Figure 1a and c). To test this hypothesis, ALK2 p.His286Asp was examined for its capacity to induce BMPspecific transcriptional reporter activity. ${ }^{19}$ In this reporter assay, the ALK2 p.His286Asp variant showed significantly lower luciferase

Table 1 Predictions of ALK3 and ErbB3 variant using PolyPhen and SIFT

\begin{tabular}{lll}
\hline & PolyPhen & SIFT \\
\hline ALK3 p.Glu415Lys & Benign variant & Affect protein function \\
ErbB3 p.Thr1169lle & Benign variant & Alter protein function (low confidence)
\end{tabular}

activity with and without BMP6 induction compared with wt ALK2 $(P<0.05)$ (Figure 2a). Next, endogenous ALK2 expression was knocked down by an RNAi construct as previously described. ${ }^{14}$ Transfection with the ALK2 p.His286Asp variant demonstrated significantly lower levels of luciferase activity in the uninduced state $(P<0.05)$, but a difference from wt ALK2 was not observed upon stimulation with BMP6 (Figure 2b). These results indicate that the ALK2 p.His286Asp receptor is capable of propagating a signal when stimulated with a BMP ligand, but this activity is impaired when the ALK2 p.His286Asp variant is in the presence of the wt ALK2 form of the receptor.

To investigate the effect of the ALK2 p.His286Asp variant on receptor function in vivo, synthetic wt ALK2 and ALK2 p.His286Asp mRNAs were injected into zebrafish embryos at the single-cell stage. Injection of the ALK2 p.His286Asp variant resulted in mild dorsalization of the zebrafish (C1 in 17 versus 3\% of injection with wt ALK2; $P<0.05),{ }^{24}$ which demonstrates a mild dominant-interfering effect of the ALK2 p.His286Asp variant on BMP signaling in the gastrulating
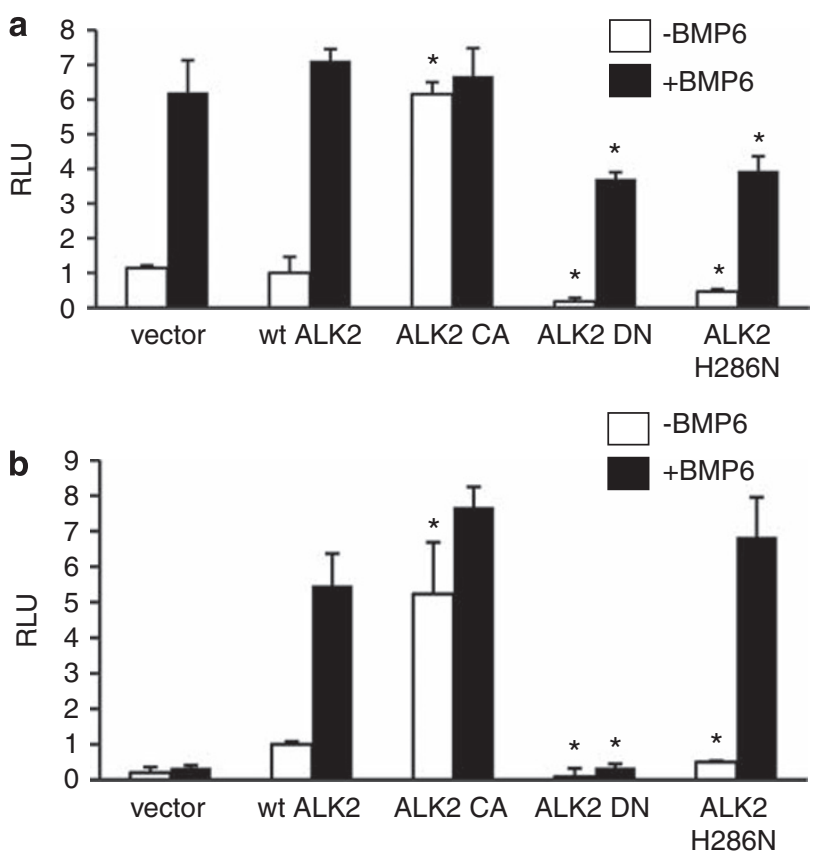

Figure 2 Representative luciferase assay on bovine aortic endothelial (BAE) cells transiently transfected with a BMP-responsive element fused to luciferase, renilla (to control the transfection) and ALK2 constructs. ALK2 activity was measured as relative luciferase units (RLU), without (white) and with (black) induction by BMP6 protein. (a) Significantly higher RLU was observed for ALK2 constitutively active (CA) without induction, whereas significantly lower RLU was observed for ALK2 dominant negative (DN) with and without BMP6 induction. For the p.His286Asp variant, both without and with induction with BMP6 significantly lower RLU was observed. (b) Luciferase assay on BAE cells as described above, including transfection of the bovine ALK2 RNAi construct to knockdown endogenous bovine ALK2 but not vector-based human ALK2. A similar pattern for ALK2 CA, ALK2 DN and wt ALK2 was observed as with injection of vector only. However, transfection with the p.His286Asp variant demonstrated significantly lower RLU without BMP6 induction, whereas induction with BMP6 did not demonstrate a significant difference between wt ALK2 and the p.His286Asp variant. RLU were measured as luciferase activity/renilla activity and expressed relative to unstimulated wt ALK2 activity. RLU are depicted as mean \pm SEM, where $n=5$ (3 replicates per experiment). Statistical significance, compared with wt ALK2, was determined by $t$-test, where ${ }^{*} P<0.05$ 

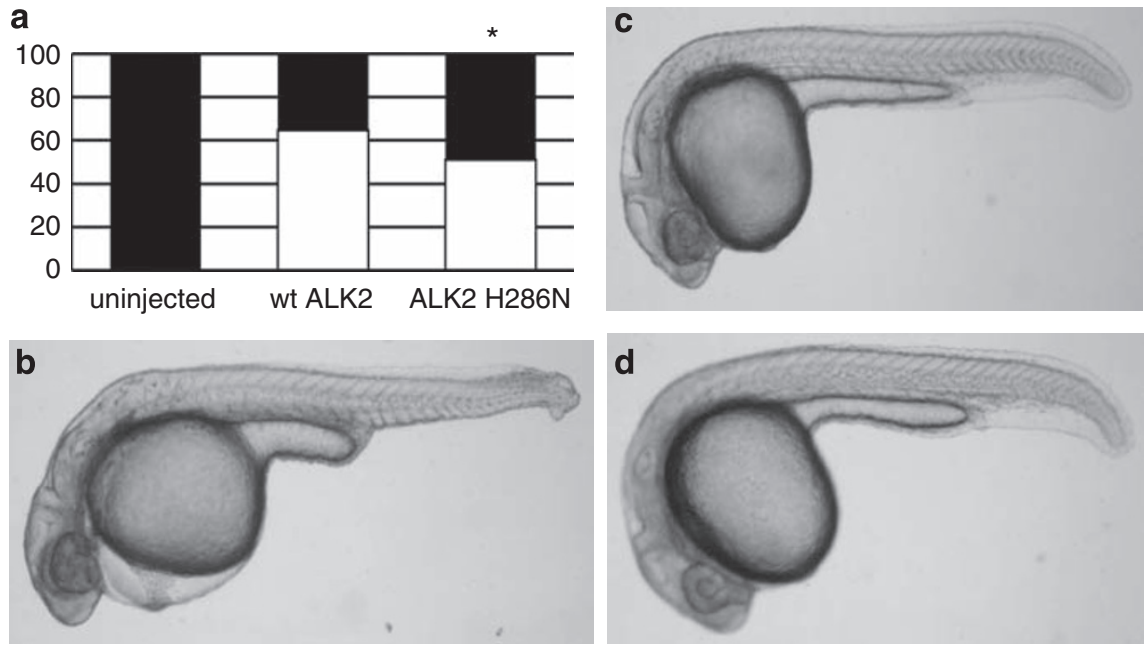

Figure 3 Rescue experiments in laf mutants. Rescue experiments in the zebrafish alk2/8 mutant, lost-a-fin (laf). (a) Injection of wt ALK2 or p.His286Asp RNA at the single-cell stage in laf/Alk2 mutants. Wt ALK2 RNA rescues phenotype in 65\% of mutant embryos, while injection of p.His 286 Asp rescues the phenotype in $51 \%$ of mutant embryos. This is significantly less effective compared with wt RNA injection. Statistical significance was determined by Student's $t$-test, where ${ }^{*} P<0.05$. (b) laf embryo identifiable by its $\mathrm{C} 1$ dorsalization phenotype, with cardiac edema and absent ventral fin. (c) Rescued laf zebrafish sibling after injection with ALK2 p.His286Asp demonstrating wt phenotype. (d) wt zebrafish.

embryo (Figure 3a). As a further measure of the reduction of ALK2 signaling for the ALK2 p.His286Asp variant, rescue experiments were performed in the zebrafish ALK2/8 mutant, lost-a-fin (laf) which is identifiable for its $\mathrm{C} 1$ dorsalization phenotype (Figure 3c). Although both wt ALK2 and variant ALK2 mRNA were able to rescue the C1 dorsalization phenotype in laf/alk2 embryos, the ALK2 p.His286Asp did so less effectively than wt (51 versus $65 \%$, respectively; $P<0.05$; Figure 3a).

\section{DISCUSSION}

We recently reported a dominant-negative $A L K 2$ allele in a patient with a CHD. ${ }^{14}$ In the current study, we report on a patient with DS and a primum-type atrial septal defect who harbors three cSNPs in genes associated with endocardial cushion development. The cSNPs result in missense substitutions in the type I BMP receptor genes, $A L K 2$ and $A L K 3$, resulting in the genetic variants p.His286Asp and p.Glu414Lys, respectively, and in the epidermal growth factor receptor family gene, ERRB3, resulting in the p.Thr1169Ile variant.

We show that, although subtle, there is a statistically significant difference in BMP signaling activity in ALK2 p.His286Asp compared with the wt ALK2 allele by both in vitro and in vivo analysis. Additionally, a genetic variant in $A L K 3$ was identified. How these compound substitutions in two distinct type I BMP receptors affect overall BMP signaling was not tested here, but could affect BMP signaling to a greater extent when present in a compound manner, because ALK2 and ALK3 can form a heterodimeric complex. ${ }^{25}$ Furthermore, it is difficult to predict what adverse effects may arise when occurring in concert with the non-conservative ERBB3 p.Thr1169Ile substitution. What can be determined from the family data is that none of these variants are sufficient to cause a CHD when present in isolation in the absence of trisomy 21 .

The three variants were detected in the parents of the probands, demonstrating that these variants were not acquired de novo. The father of the proband carries the ALK2 p.His286Asp and ALK3 p.Glu414Lys variants, and the sister of the proband harbors the ALK3 p.Glu414Lys and the ERBB3 p.Thr1169Ile variants. Neither of these individuals presented with a CHD, demonstrating that the possession of either of these two combinations of cSNPs is either not sufficient to cause the heart defect or the variants are not completely penetrant.

Our data are consistent with the notion that $A L K 2$ is a susceptibility gene for primum-type atrial septal defects, in combination with variants at other loci or in combination with trisomy 21 . This case is also suggestive that additional predisposing genetic factors exist for heart defects in DS patients, thus underlining the polygenic nature of these defects. These genetic factors can be identified by large-scale candidate gene screens.

\section{CONFLICT OF INTEREST}

The authors declare no conflict of interest.

\section{ACKNOWLEDGEMENTS}

We kindly acknowledge the cooperation of the index patient and his family members. Work in JB's laboratory was partly supported by EU FP6 grant LSHM-CT-2005-018833, EUGeneHeart.

1 Bell R, Rankin J, Donaldson LJ: Down's syndrome: occurrence and outcome in the north of England, 1985-99. Paediatr Perinat Epidemiol 2003; 17: 33-39.

2 Mikkelsen M, Fischer G, Stene J, Stene E, Petersen E: Incidence study of Down's syndrome in Copenhagen, 1960-1971; with chromosome investigation. Ann Hum Genet 1976; 40: 177-182.

3 Owens JR, Harris F, Walker S, McAllister E, West L: The incidence of Down's syndrome over a 19-year period with special reference to maternal age. J Med Genet 1983; 20: 90-93.

4 Korenberg JR, Chen XN, Schipper R et al: Down syndrome phenotypes: the consequences of chromosomal imbalance. Proc Natl Acad Sci USA 1994; 91: 4997-5001.

5 Wiseman FK, Alford KA, Tybulewicz VL, Fisher EM: Down syndrome-recent progress and future prospects. Hum Mol Genet 2009; 18: R75-R83.

6 Loffredo CA, Hirata J, Wilson PD, Ferencz C, Lurie IW: Atrioventricular septal defects: possible etiologic differences between complete and partial defects. Teratology 2001; 63: 87-93.

7 Paladini D, Tartaglione A, Agangi A et al: The association between congenital heart disease and Down syndrome in prenatal life. Ultrasound Obstet Gynecol 2000; 15: 104-108.

8 Vis JC, Duffels MG, Winter MM et al: Down syndrome: a cardiovascular perspective. J Intellect Disabil Res 2009; 53: 419-425.

9 Korenberg JR, Kawashima H, Pulst SM et al: Molecular definition of a region of chromosome 21 that causes features of the Down syndrome phenotype. Am J Hum Genet 1990; 47: 236-246. 
10 Kerstann KF, Feingold E, Freeman SB et al: Linkage disequilibrium mapping in trisomic populations: analytical approaches and an application to congenital heart defects in Down syndrome. Genet Epidemiol 2004; 27: 240-251.

11 Klewer SE, Krob SL, Kolker SJ, Kitten GT: Expression of type VI collagen in the developing mouse heart. Dev Dyn 1998; 211: 248-255.

12 Gittenberger-de Groot AC, Bartram U, Oosthoek PW et al: Collagen type VI expression during cardiac development and in human fetuses with trisomy 21. Anat Rec A Discov Mol Cell Evol Biol 2003; 275: 1109-1116.

13 Maslen CL, Babcock D, Robinson SW et al: CRELD1 mutations contribute to the occurrence of cardiac atrioventricular septal defects in Down syndrome. Am J Med Genet A 2006; 140: 2501-2505.

14 Smith KA, Joziasse IC, Chocron S et al: Identification of a dominant-negative ALK2 allele in a family with congenital heart defects. Circulation 2009; 119 3062-3069.

15 van der Velde ET, Vriend JW, Mannens MM et al: CONCOR, an initiative towards a national registry and DNA-bank of patients with congenital heart disease in the Netherlands: rationale, design, and first results. Eur J Epidemiol 2005; 20 : 549-557.

16 Prandini P, Deutsch S, Lyle R et al: Natural gene-expression variation in Down syndrome modulates the outcome of gene-dosage imbalance. Am J Hum Genet 2007; 81: 252-263.
17 Hammerschmidt M, Mullins MC: Dorsoventral patterning in the zebrafish: bone morphogenetic proteins and beyond. Results Probl Cell Differ 2002; 40: 72-95.

18 ten Dijke $\mathrm{P}$, Yamashita $\mathrm{H}$, Ichijo $\mathrm{H}$ et al: Characterization of type I receptors for transforming growth factor-beta and activin. Science 1994; 264: 101-104.

19 Korchynskyi O, ten Dijke P: Identification and functional characterization of distinct critically important bone morphogenetic protein-specific response elements in the Id1 promoter. J Biol Chem 2002; 277: 4883-4891.

20 Westerfield M: The Zebrafish Book. A Guide for the Laboratory Use of Zebrafish (Danio rerio), 4th edn. Eugene: University of Oregon Press, 2000.

21 Sunyaev S, Ramensky V, Bork P: Towards a structural basis of human non-synonymous single nucleotide polymorphisms. Trends Genet 2000; 16: 198-200.

22 Sunyaev S, Ramensky V, Koch I, Lathe III W, Kondrashov AS, Bork P: Prediction of deleterious human alleles. Hum Mol Genet 2001; 10: 591-597.

$23 \mathrm{Ng}$ PC, Henikoff S: SIFT: predicting amino acid changes that affect protein function. Nucleic Acids Res 2003; 31: 3812-3814.

24 Mullins MC, Hammerschmidt M, Kane DA et al: Genes establishing dorsoventral pattern formation in the zebrafish embryo: the ventral specifying genes. Development 1996; 123: 81-93.

25 Little SC, Mullins MC: Bone morphogenetic protein heterodimers assemble heteromeric type I receptor complexes to pattern the dorsoventral axis. Nat Cell Biol 2009; 11: 637-643.

Supplementary Information accompanies the paper on European Journal of Human Genetics website (http://www.nature.com/ejhg) 